\title{
A Risk Reduction Technique for Five Invasive Procedures in the Emergency Room
}

2 Using a Compact and Lightweight X-ray Unit

3

4

5

6

7
Yutaka Igarashi $^{1 *}$, Shimpei Ikeda ${ }^{1,2}$, Kunio Hirai $^{3}$, Naoki Tominaga ${ }^{1,4}$, Taiki Mizobuchi $^{1}$, Kenta Shigeta ${ }^{1}$, Hiromoto Ishii ${ }^{1}$, Shoji Yokobori ${ }^{1}$

1. Department of Emergency and Critical Care Medicine, Nippon Medical School 1-1-5 Sendagi, Bunkyo, Tokyo, 113-8603, Japan

2. Department of Radiology, Nippon Medical School Chiba Hokusoh Hospital 1715 Kamagari, Inzai, Chiba 270-1694, Japan

3. Division of Radiological Technology, Nippon Medical School Hospital 1-1-5 Sendagi, Bunkyo, Tokyo, 113-8603, Japan

4. Department of Emergency and Critical Care Medicine, Saitama City Hospital 2460, Mimuro, Midori, Saitama, 336-8522, Japan

*Corresponding author

Yutaka Igarashi

Department of Emergency and Critical Care Medicine

Nippon Medical School

1-1-5 Sendagi, Bunkyo-ku, Tokyo, 113-8603, Japan

TEL +81-3-3822-2131

FAX +81-3-3821-5102

E-MAIL igarashiy@nms.ac.jp

Short title: Portable X-ray and invasive procedures

Keywords: Thoracic radiography; Complications; Central venous catheters, Chest tubes; Tracheotomy 


\section{Abstract}

34 Background: Many invasive procedures are performed in the emergency room (ER),

35 which have potential risks and complications. Due to limitations, especially with respect

36 to size, portable X-ray devices are generally not used during such procedures. However,

37 they have been miniaturized, enabling physicians to capture X-ray images by

38 themselves..

39 Methods: We developed a safe, compact, and lightweight X-ray unit and performed five

40 invasive procedures in the ER. In all the procedures, a chest X-ray image was taken to

41 confirm its utility.

42 Results: Case 1 (central venous catheter placement): After needle and guidewire

43 insertion and the placement of the catheter, the location of catheter could be confirmed.

44 Case 2 (chest tube insertion): During the insertion of the chest tube into the pleural

45 space, it was observed that the tip of the thoracic tube was at the appropriate location.

46 Case 3 (percutaneous tracheostomy or cricothyroidotomy): After needle and guidewire

47 insertion, it was visualized that the guidewire was in the right main bronchus and that

48 the tube was inserted into the trachea. Case 4 (resuscitative endovascular aortic balloon

49 of the aorta): The captured image revealed that the catheter was located in zone I before 
50 balloon inflation. Case 5 (Sengstaken-Blakemore tube): The image revealed that the

51 balloon was located in the stomach.

52 Conclusions: The devised portable X-ray unit could contribute medical safety during

53 invasive procedures frequently performed in the ER.

54

55 


\section{Introduction}

57 Critically ill patients, regardless of the cause, are first transferred to the emergency room

58 (ER) for diagnosis and resuscitation. Many invasive procedures performed for such

59 patients in the ER are associated with potential risks as they could be the cause for post

60 procedural complications. ${ }^{1,2}$ Intraprocedural imaging such as ultrasonography and

61 fluoroscopy have been used to reduce some of the complications. However, the patients

62 must be transported to the fluoroscopy room. In addition, as ultrasound wave get disrupted

63 by air/gas and do not penetrate deep, certain areas cannot be visualized. Although

64 intraprocedural radiology does not require the patient to be moved and can capture images

65 from area that cannot be visualized with ultrasound, its use has been limited because of

66 the conventional instrument size and reliance on technicians. ${ }^{3}$

67 Over the years, portable X-ray devices have become miniaturized, permitting physicians

68 to take X-ray images anywhere with ease ${ }^{4-6}$. Thus, intraprocedural X-ray devices offer

69 safety benefits in medical procedures. However, little is known about the use of portable

70 X-ray devices during invasive procedures. Having developed and assessed the safety of

71 one such compact and lightweight X-ray unit, we, herein report its utility for frequently

72 performed invasive procedures in the ER. 


\section{$74 \quad$ Materials and Methods}

$75 \quad$ Setting

76 This study was conducted in a single institution and approved by the Ethics committee

77 of Nippon Medical School Hospital, Japan (30-09-993). It may be noted that in Japan,

78 only doctors and radiology technicians are authorized to administer X-rays.

79

80 Medical equipment

81 A compact and lightweight X-ray unit developed has been commercialized and comprises

82 of the following three parts: an X-ray tube, weighing only $3.5 \mathrm{~kg}$ (CALNEO Xair,

83 Fujifilm Corporation, Tokyo, Japan); a laptop through which the operator controls the

84 functioning of machine; and a flat panel (CALNEO Smart, Fujifilm Corporation, Tokyo,

85 Japan) (Fig. 1). Depending on the body part being examined, the following three

86 parameters were preset: the chest $(90 \mathrm{kV}, 0.5 \mathrm{mAs})$, abdomen $(90 \mathrm{kV}, 2.5 \mathrm{mAs})$, and

87 extremities $(70 \mathrm{kV}, 2 \mathrm{mAs})$. Although conventional mobile X-ray units require

88 knowledge like $\mathrm{kV}$ and $\mathrm{mAs}$ settings before examination, this device is superior in terms

89 of usability. This device has received approval from the Pharmaceuticals and Medical

90 Devices Agency, Japan, for marketing. 


\section{Procedures}

93 In this study, chest radiographs were taken to confirm the location of the catheter or

94 tube tips during five invasive procedures frequently preformed in the ER. These were

95 central venous catheter (CVC) (or peripherally inserted central catheters) placement,

96 chest tube insertion, percutaneous tracheotomy (or cricothyroidotomy), resuscitative

97 endovascular balloon occlusion of the aorta (REBOA), and Sengstaken-Blakemore

98 (SB) tube insertion.

99 While performing the procedure, the physician wearing an X-ray radiation-protective

100 apron, placed the flat panel detector of the unit under the patient and the X-ray tube

101 above. When the guidewire, catheter, or tube was inserted, the physician selected the

102 radiation intensity, pressed the relevant button, and immediately viewed the image on

103 the laptop screen. If the catheter and tube tips were malpositioned, another X-ray image

104 was taken after repositioning.

105

106 Results

107 Case 1: CVC placement

108 A 48-year-old woman was intubated and received mechanical ventilation after surgical

109 clipping for a cerebral aneurysm. The patient required CVC replacement in the right 
110 internal jugular vein. A physician set the X-ray devices, an ultrasound device, and a flat

111 panel detector under the patient as a part of preoperative preparation (Fig. 2). When the

112 needle and guidewire were inserted, a chest radiograph showed a guidewire in the

113 superior vena cava (Fig. 3a). When the catheter was placed, a chest radiograph showed

114 that the catheter tip was in the appropriate location (Fig. 3b).

116 Case 2: Chest tube insertion

117 A 70-year-old woman presented with difficulty breathing. She was diagnosed with

118 congestive heart failure and bilateral pleural effusion. The effusion was drained because

119 oxygen was required to maintain a percutaneous saturation of over $90 \%$. During the

120 insertion of the chest tube into the pleural space, a chest radiograph was taken, which

121 showed that the tip of the thoracic tube had reached the appropriate location (Fig. 4).

123 Case 3: Percutaneous tracheostomy

124 A 60-year-old man diagnosed with intracerebral hematoma was intubated and received

125 mechanical ventilation. A tracheostomy was required for prolonged endotracheal

126 intubation. A Pean clamp forceps was placed at the insertion site, and a chest radiograph

127 was taken, with the forceps determining the position of the tip of the intubation tube in 
128 relation to the insertion site (Fig. 5a). The intubation tube was withdrawn $3.5 \mathrm{~cm}$

129 because there was a $3-\mathrm{cm}$ gap between the tip of the intubation tube and the insertion

130 site (Fig. 5b). When the needle and guidewire were inserted, the chest radiograph

131 showed the guidewire in the right main bronchus (Fig. 5c). After adequate dilatation,

132 the dilator was removed, and a tracheostomy tube was inserted into the trachea above

133 the guiding catheter.

134

135 Case 4: REBOA

136 A 70-year-old man was transported to the ER after a road accident. Computed

137 tomography (CT) revealed splenic and renal hemorrhage, as well as a pelvic fracture.

138 The pateints's blood pressure decreased after the CT scan. A REBOA catheter was

139 inserted and advanced into the aorta; following which a 7-French introducer sheath was

140 inserted through the right femoral artery. A chest radiography revealed the REBOA

141 catheter in zone I, extending from the origin of the left subclavian artery to the celiac

142 artery before balloon inflation (Fig. 6). After balloon inflation, the patient's blood

143 pressure increased, and splenectomy could be performed. 
146 A 48-year-old man diagnosed with alcoholic cirrhosis and esophageal varices presented

147 with hematemesis and was transferred to our hospital. He presented with hypotension

148 and anemia with a hemoglobin level of $3.1 \mathrm{~g} / \mathrm{dL}$. As it was difficult to stop the bleeding

149 with an endoscope, an SB tube was inserted. Following balloon inflation, a chest

150 radiograph revealed that the balloon was located in the stomach (Fig. 7). The SB tube

151 was then pulled, and the esophageal balloon was inflated. Subsequently, the blood

152 pressure increased and the hemoglobin level normalized. The next day, the endoscopic

153 variceal ligation was performed successfully.

154

\section{Discussion}

156 We developed an intraprocedural visualization technique which uses a compact and

157 lightweight X-ray unit in five frequently performed invasive procedures. Based on the

158 observations from these case studies, it can be implied that this technique can reduce the

159 risk of complications during invasive procedures.

160 We reviewed the complications of each procedure and described how a compact and

161 lightweight X-ray unit are useful for preventing complications. 
163 CVC insertion: Procedures for CVC insertion have progressed in terms of safety and

164 ease of performance. However, the complication rate of CVC insertion continues to

165 remain high. A retrospective cohort study described an overall complication rate of

$16615 \%$. The complications included failure to place the catheter (22\%), arterial puncture

167 (5\%), catheter malposition (4\%), pneumothorax (1\%), subcutaneous hematoma (1\%),

168 hemothorax $(<1 \%)$, and cardiac arrest $(<1 \%)$. Ultrasound-guided techniques have

169 reduced the risk of inadvertent arterial puncture (risk ratio [RR] 0.21, 95\% confidence

170 interval $[\mathrm{CI}] 0.06$ to $0.82 ; P=0.02)$ and hematoma (RR $0.26,95 \%$ CI 0.09 to $0.76 ; P=$

171 0.01) for the subclavian vein. However, ultrasound-guided techniques cannot detect

172 misplacement of the CVC or ensure placement at the appropriate location. Fluoroscopy

173 is often used for insertion of peripherally inserted central catheter and a randomized

174 controlled trial revealed that mispositioning of catheter tip was significantly lower in

175 fluoroscopy guided technique compared with blind bedside technique ${ }^{7}$. An X-ray

176 guided technique, on the contrary, might avoid arrhythmia that can be induced by

177 inserting a guidewire and catheter too deeply. ${ }^{8}$ Moreover, using this technique,

178 physicians can spot any abnormal findings even in case of an iatrogenic pneumothorax. 
180 Chest tube insertion: Chest tube drains are indicated in case of a hemothorax, massive

181 pleural effusion, large or progressive pneumothorax, and postoperatively in thoracic

182 surgery. All intrathoracic organs, including the lung, diaphragm, heart, large vessels,

183 and esophagus, are potentially at a risk of injury during the chest tube insertion. A chest

184 tube placed in the right ventricle, though rare, is life-threatening Early $(<24 \mathrm{~h}$ after

185 placement) complications occurred in $3 \%$, and $8 \%$ of cases have been reported. ${ }^{9}$

186 Although point-of-care ultrasound during tube insertion has been used to reduce

187 complications, ${ }^{10}$ it cannot confirm the placement of the tip of the chest tube, which

188 could be placed at an inappropriate location such as within the interlobar space.

189 Appropriate placement of the tip of the chest tube can be confirmed using a compact

190 and lightweight X-ray unit.

192 Percutaneous tracheostomy (or cricothyroidotomy): The technique of percutaneous

193 tracheostomy was developed in 1985, based on modification of the Seldinger principles.

194 The advantages of this technique as compared with surgical tracheostomy are

195 simplicity, a smaller skin incision, lower incidence of wound infections and peristomal

196 bleeding, and decreased mortality rates associated with patient transfer. ${ }^{11-15}$ However,

197 perioperative complications of percutaneous tracheostomy include paratracheal 
198 insertion, tracheal laceration, pneumothorax, loss of airway, and hemorrhage. ${ }^{16}$ An X-

199 ray guided technique could assist confirming the location of the tube and guidewire

200 during the procedure and paratracheal insertion, thereby reducing the possibility of

201 developing a tracheoesophageal fistula. Additionally, though bronchoscopy might be

202 helpful for percutaneous tracheostomy ${ }^{17}$, it occupies more space and requires an

203 operator too. Hence the compact and lightweight X-ray unit could replace a

204 bronchoscope. Moreover, our technique can be applied not only in percutaneous

205 tracheostomy but also in cricothyroidotomy.

207 REBOA: REBOA is an alternative to aortic clamping by thoracotomy for non-

208 compressible torso hemorrhage and is a tool for temporary hemostasis. Confirming the

209 position of the REBOA catheter when inflated by fluoroscopy or X-ray imaging is

210 recommended. The actual and theoretical risks include unintended placement in the

211 aortic arch, renal artery, zone II, or contralateral iliac artery. ${ }^{18}$ A number of

212 complications, such as aortic injury, ischemic injury, arterial dissection, thrombosis, and

213 embolic events, have been reported. ${ }^{19,20}$ The appropriate placement of the tip of the

214 REBOA catheter can thus be confirmed using the compact and lightweight X-ray unit. 
$216 S B$ tube: Many major complications caused by inflation of the gastric balloon outside

217 the stomach have been reported, including airway obstruction, tracheal injury,

218 esophageal rupture, and jejunal rupture. ${ }^{21-26}$ Although the position can be confirmed by

219 auscultation, the manufacturer recommends the use of fluoroscopy. However, rupture of

220 the esophageal varices often results in shock due to massive bleeding, and the patient

221 may have difficulty in moving. Hence, the compact and lightweight X-ray unit may be

222 useful in this procedure too.

224 Time reduction

225 This device has several potential advantages. Physicians can take X-ray images by

226 themselves as soon as the patient is presented to the hospital and save at least the travel

227 time compared with technician. If physician A technician would take on average 12.4

$228 \mathrm{~min}$ from being informed to get the portable X-ray unit and $22.7 \mathrm{~min}$ to upload images

229 and archive them in the communication systems ${ }^{27}$. In addition, though fluoroscopy-

230 guided procedures can prevent complications, they require transferring the patient to the

231 fluoroscopy room. ${ }^{7}$ According to a study of peripherally inserted central catheters, the

232 average transfer time was approximately $26 \mathrm{~min}$, which was similar to the procedure 
233 time. ${ }^{8}$ Our technique could be particularly useful for critically ill patients who cannot be

234 transferred safely to the fluoroscopy room.

236 Radiation dose

237 A single chest $\mathrm{X}$-ray causes an exposure to $0.02 \mathrm{mGy}$ of radiation ${ }^{5}$ whereas fluoroscopy 238 causes $20 \mathrm{mGy} / \mathrm{min}$ of radiation ${ }^{28}$. A total of $0.63 \mathrm{~min}$ is required to insert a CVC by 239 radiologists using fluoroscopy, ${ }^{29}$ and patients are exposed to an average of $12 \mathrm{mGy}$ of

240 radiation with fluoroscopy during CVC insertion. Therefore, a compact X-ray technique

241 would reduce the lower radiation exposure to patients during CVC insertion as compared with fluoroscopy. ${ }^{29}$

244 Limitations

245 This study has several limitations. First, no control group was included in this study.

246 Although we think that visible is safer than invisible, it could not be evaluated how the

247 device would contribute to safety. As far as we know, no trouble has been caused by

248 this method, but there is a possibility that some trouble may occur. There is insufficient

249 consideration of risk assessment and trouble shooting. Second, the study did not

250 evaluate image quality. No X-ray images were retaken because of poor quality. 
251 Emergency physicians are not familiar with adjusting the position of the flat panel

252 detector. Hence, at times, the image could not be captured. It would probably more

253 appropriate to use a larger panel to resolve this issue.

254

255 Conclusions

256 We have introduced a visualization technique for invasive procedures using a compact

257 and lightweight X-ray unit in the ER. It can be implied that this technique can reduce

258 the risk of complications during invasive procedures. 


\section{Abbreviations}

260 CI, confidence interval

261 CT, computed tomography

262 CVC, central venous catheter

263 ER, emergency room

264 REBOA, resuscitative endovascular balloon occlusion of the aorta

265 RR, risk ratio

266 SB, Sengstaken-Blakemore

267 


\section{References}

270 1. Comerlato PH, Rebelatto TF, Santiago de Almeida FA, et al. Complications of central venous catheter insertion in a teaching hospital. Rev Assoc Med Bras (1992). 2017 Jul;63(7):613-20. Available from: https://www.ncbi.nlm.nih.gov/pubmed/28977087.

2. Silberzweig JE, Sacks D, Khorsandi AS, Bakal CW, Society of Interventional

3. Shah R, Qayed E. Bedside Endoscopic Retrograde Cholangiopancreatography Using Portable X-Ray in Acute Severe Cholangitis. Case Rep Gastrointest Med. 2018;2018:8763671. Available from: https://www.ncbi.nlm.nih.gov/pubmed/29682365.

4. Omori K, Muramatsu KI, Nagasawa H, et al. The Utility of a Portable X-ray System. Air Med J. 2019 May - Jun;38(3):212-4. Available from: https://www.ncbi.nlm.nih.gov/pubmed/31122590.

5. Omori K, Yanagawa Y, Muramatsu KI, et al. Experience using a portable X-ray system at the scene transported by a physician-staffed helicopter. Acute Med Surg. 2019 Oct;6(4):396-9. Available from: https://www.ncbi.nlm.nih.gov/pubmed/31592083.

6. Yanagawa Y, Ohsaka H, Oode Y, Omori K. A case of fatal trauma evaluated using a portable X-ray system at the scene. J Rural Med. 2019 Nov;14(2):249-52. Available from: https://www.ncbi.nlm.nih.gov/pubmed/31788152.

7. Glauser F, Breault S, Rigamonti F, Sotiriadis C, Jouannic AM, Qanadli SD. Tip malposition of peripherally inserted central catheters: a prospective randomized controlled trial to compare bedside insertion to fluoroscopically guided placement. Eur Radiol. $2017 \quad$ Jul;27(7):2843-9. Available from: https://www.ncbi.nlm.nih.gov/pubmed/27957644.

8. Cho SB, Baek HJ, Park SE, et al. Clinical feasibility and effectiveness of bedside peripherally inserted central catheter using portable digital radiography for patients in an intensive care unit: A single-center experience. Medicine. 2019 Jun;98(26):e16197. Available from: https://www.ncbi.nlm.nih.gov/pubmed/31261562.

9. Collop NA, Kim S, Sahn SA. Analysis of tube thoracostomy performed by 
pulmonologists at a teaching hospital. Chest. 1997 Sep;112(3):709-13. Available from: https://www.ncbi.nlm.nih.gov/pubmed/9315804.

10. McElnay PJ, Lim E. Modern Techniques to Insert Chest Drains. Thorac Surg Clin. 2017

Feb;27(1):29-34.

Available

from:

https://www.ncbi.nlm.nih.gov/pubmed/27865324.

11. Friedman Y, Mayer AD. Bedside percutaneous tracheostomy in critically ill patients. Chest. 1993 Aug;104(2):532-5. Available from: https://www.ncbi.nlm.nih.gov/pubmed/8339644.

12. Toye FJ, Weinstein JD. Clinical experience with percutaneous tracheostomy and cricothyroidotomy in 100 patients. J Trauma. 1986 Nov;26(11):1034-40. Available from: https://www.ncbi.nlm.nih.gov/pubmed/3783779.

13. Hazard P, Jones C, Benitone J. Comparative clinical trial of standard operative tracheostomy with percutaneous tracheostomy. Crit Care Med. 1991 Aug;19(8):1018-24.

Available

from: https://www.ncbi.nlm.nih.gov/pubmed/1860325.

14. Griggs WM, Myburgh JA, Worthley LI. A prospective comparison of a percutaneous tracheostomy technique with standard surgical tracheostomy. Intensive Care Med. 1991;17(5):261-3. Available from: https://www.ncbi.nlm.nih.gov/pubmed/1939869.

15. Ciaglia P, Graniero KD. Percutaneous dilatational tracheostomy. Results and longterm follow-up. Chest. 1992 Feb;101(2):464-7. Available from: https://www.ncbi.nlm.nih.gov/pubmed/1735273.

16. Klotz R, Probst P, Deininger M, et al. Percutaneous versus surgical strategy for tracheostomy: a systematic review and meta-analysis of perioperative and postoperative complications. Langenbecks Arch Surg. 2018 Mar;403(2):137-49. Available from: https://www.ncbi.nlm.nih.gov/pubmed/29282535.

17. Easterday TS, Moore JW, Redden MH, et al. Percutaneous Tracheostomy under Bronchoscopic Visualization Does Not Affect Short-Term or Long-Term Complications. The American surgeon. 2017 Jul 1;83(7):696-8. Available from: https://www.ncbi.nlm.nih.gov/pubmed/28738937.

18. Scott DJ, Eliason JL, Villamaria C, et al. A novel fluoroscopy-free, resuscitative endovascular aortic balloon occlusion system in a model of hemorrhagic shock. J Trauma Acute Care Surg. 2013 Jul;75(1):122-8. Available from: https://www.ncbi.nlm.nih.gov/pubmed/23940855.

19. Long B, Hafen L, Koyfman A, Gottlieb M. Resuscitative Endovascular Balloon Occlusion of the Aorta: A Review for Emergency Clinicians. J Emerg Med. 2019 
https://www.ncbi.nlm.nih.gov/pubmed/31010604.

20. Ribeiro Junior MAF, Feng CYD, Nguyen ATM, et al. The complications associated with Resuscitative Endovascular Balloon Occlusion of the Aorta (REBOA). World J Emerg Surg. 2018;13:20. Available from: https://www.ncbi.nlm.nih.gov/pubmed/29774048.

21. Goff JS, Thompson JS, Pratt CF, Tomasso GI, Penn I. Jejunal rupture caused by a Sengstaken-Blakemore tube. Gastroenterology. 1982 Mar;82(3):573-5. Available from: https://www.ncbi.nlm.nih.gov/pubmed/6976285.

22. Wang HS, Lin J, Wang F, Miao L. Tracheal injury characterized by subcutaneous emphysema and dyspnea after improper placement of a Sengstaken-Blakemore tube: A case report. Medicine. 2018 Jul;97(30):e11289. Available from: https://www.ncbi.nlm.nih.gov/pubmed/30045253.

23. Hamm DD, Papp JP. Rupture of esophagus during use of Sengstaken-Blakemore tube. Postgraduate medicine. 1974 Aug;56(2):199-200. Available from: https://www.ncbi.nlm.nih.gov/pubmed/4854179.

24. Chien JY, Yu CJ. Images in clinical medicine. Malposition of a SengstakenBlakemore tube. N Engl J Med. 2005 Feb 24;352(8):e7. Available from: https://www.ncbi.nlm.nih.gov/pubmed/15728803.

25. Kelly DJ, Walsh F, Ahmed S, Synnott A. Airway obstruction due to a SengstakenBlakemore tube. Anesth Analg. 1997 Jul;85(1):219-21. Available from: https://www.ncbi.nlm.nih.gov/pubmed/9212152.

26. Nielsen TS, Charles AV. Lethal esophageal rupture following treatment with Sengstaken-Blakemore tube in management of variceal bleeding: a 10-year autopsy study. Forensic Sci Int. 2012 Oct 10;222(1-3):e19-22. Available from: https://www.ncbi.nlm.nih.gov/pubmed/22721936.

27. Abujudeh H, Vuong B, Baker SR. Quality and operations of portable X-ray examination procedures in the emergency room: queuing theory at work. Emerg Radiol. 2005 Jul;11(5):262-6. Available from: https://www.ncbi.nlm.nih.gov/pubmed/16133619.

28. Lee RK, Chu WC, Graham CA, Rainer TH, Ahuja AT. Knowledge of radiation exposure in common radiological investigations: a comparison between radiologists and non-radiologists. Emerg Med J. 2012 Apr;29(4):306-8. Available from: https://www.ncbi.nlm.nih.gov/pubmed/21873321.

29. Xu BJ, Duszak R, Jr., McGinnis RS, Stanfill JG, O'Rear J, An AQ. Increased fluoroscopy time for central venous catheter placement by radiology residents 


\section{Figure Legends}

$379 \quad$ Figure 1

380 Illustration of the compact and lightweight X-ray unit and comparison with other widely

381 used portable X-ray devices (Sirius 130 HP, Hitachi, Tokyo, Japan). The compact and

382 lightweight X-ray unit comprises three parts: an X-ray tube (CALNEO Xair, Fujifilm

383 Corporation, Tokyo, Japan), a laptop computer and a flat panel detector (CALNEO

384 Smart, Fujifilm Corporation, Tokyo, Japan).

385

$386 \quad$ Figure 2

387 Setting for the compact and lightweight X-ray unit and ultrasonography device. A

388 physician sets the X-ray unit, an ultrasound device, and a flat panel detector under the

389 patient as a part of preoperative preparation.

390

$391 \quad$ Figure 3

392 Central venous catheter placement. (A) Chest X-ray image showing the tip of the

393 guidewire (arrowhead) in the superior vena cava. (B) Chest X-ray image showing the tip

394 of catheter (arrowhead) in the superior vena cava. 
396 Figure 4

397 Chest tube insertion. Chest X-ray image showing bilateral pleural effusion. The tip of

398 the chest tube (arrowhead) is located at the left apical pulmonary lesion.

399

$400 \quad$ Figure 5

401 Percutaneous tracheostomy. (A) Image showing a distance of $3 \mathrm{~cm}$ between the tip of

402 endotracheal tube and the Pean clamp forceps. (B) Image showing the location of the tip

403 of the endotracheal tube compared with the Pean clamp forceps following the

404 withdrawal of the endotracheal tube by $3.5 \mathrm{~cm}$. (C) Chest X-ray image showing the

405 guidewire in the right main bronchus.

406

$407 \quad$ Figure 6

408 Resuscitative endovascular balloon occlusion of the aorta. Chest X-ray image showing

409 the tip of the resuscitative endovascular balloon occlusion of the aorta catheter

410 (arrowhead) in zone I.

411

412

413 


\section{$414 \quad$ Figure 7}

415 Sengstaken-Blakemore (SB) tube insertion. Chest X-ray image showing the balloon of

416 the SB tube (arrowhead) in the stomach.

417

\section{Acknowledgments}

419 This study was conducted in collaboration with Fujifilm Corporation (Tokyo, Japan),

420 which provided the medical equipment and technical support.

421 
Fig. 1

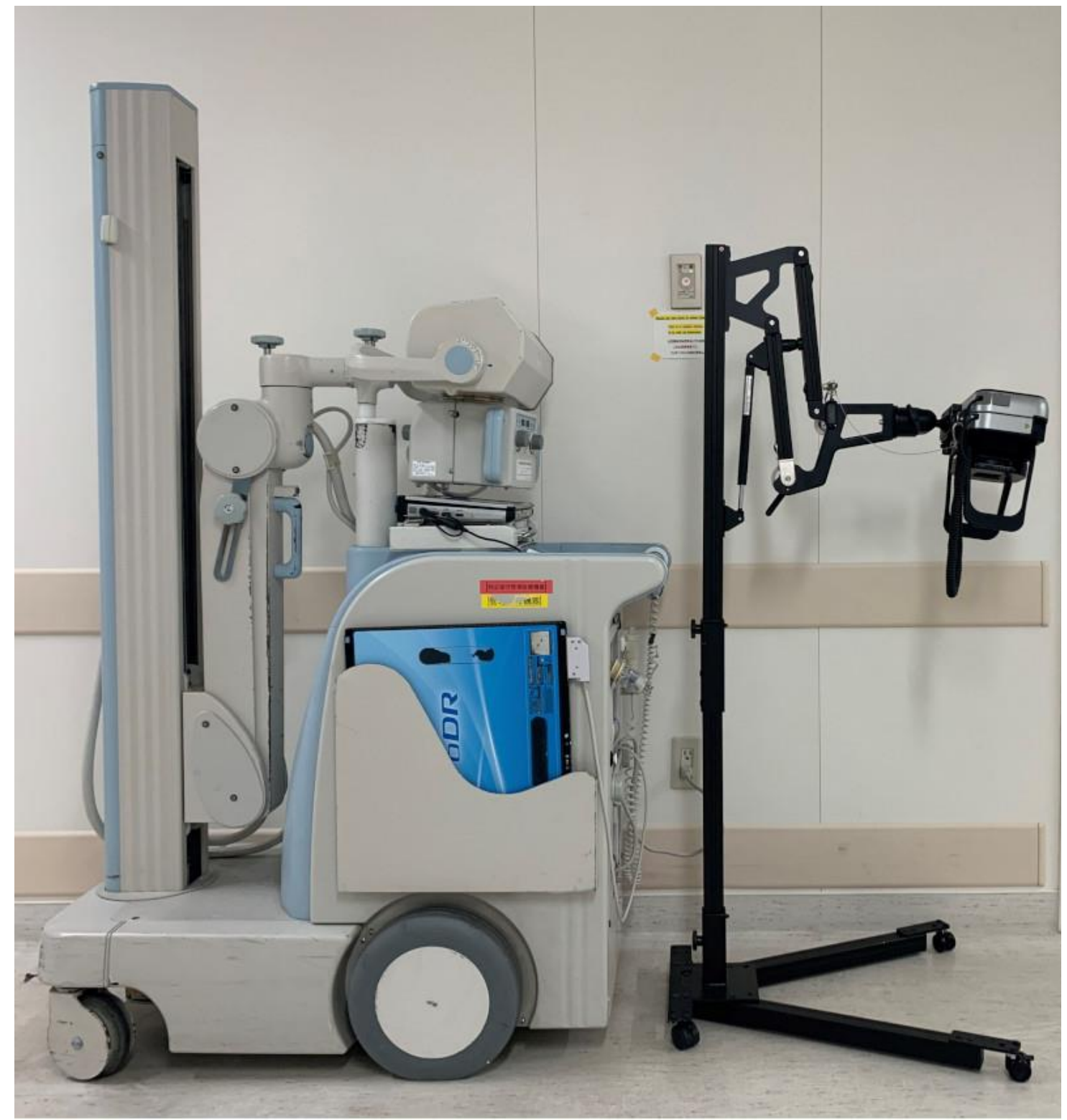


Fig. 2

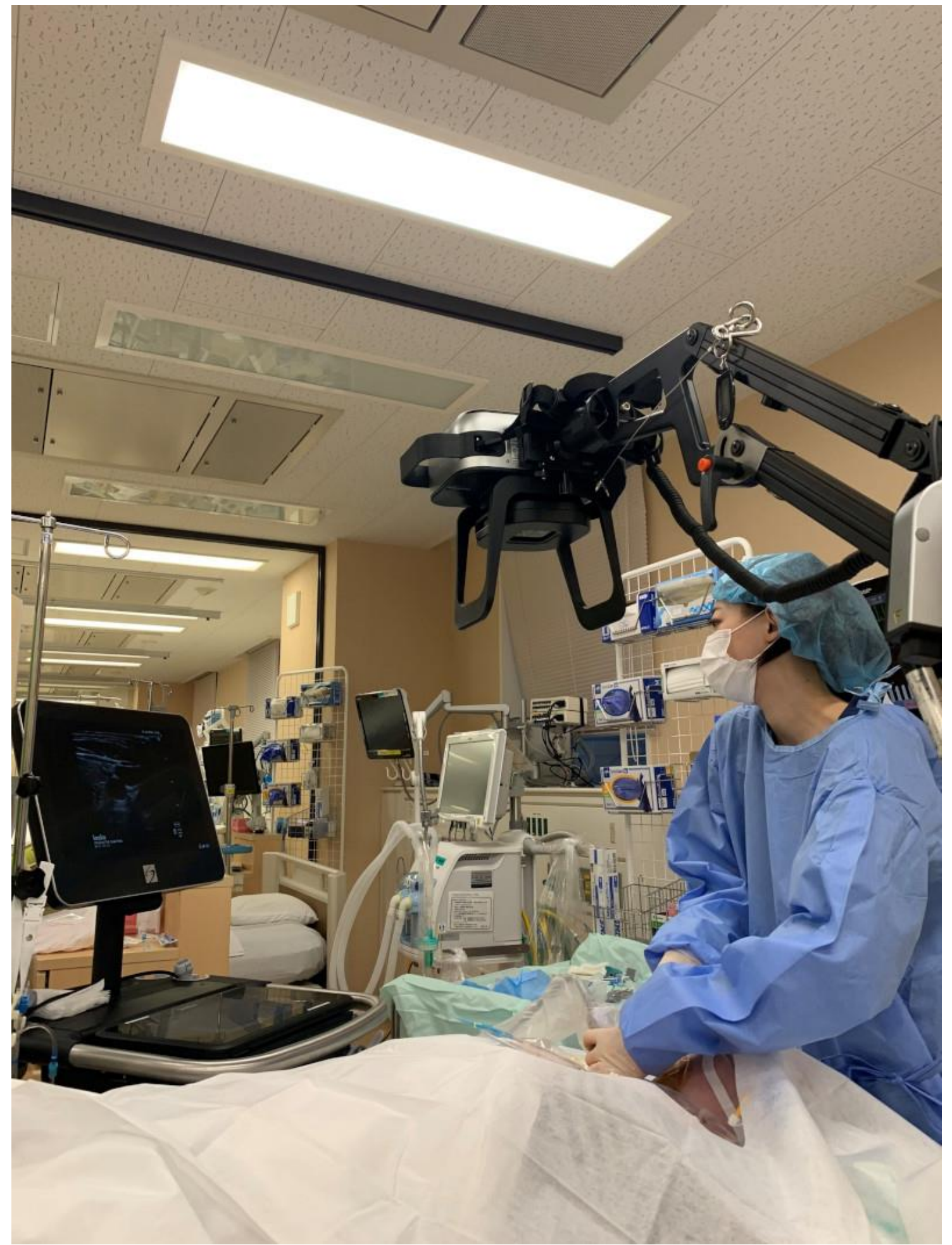


Fig. 3

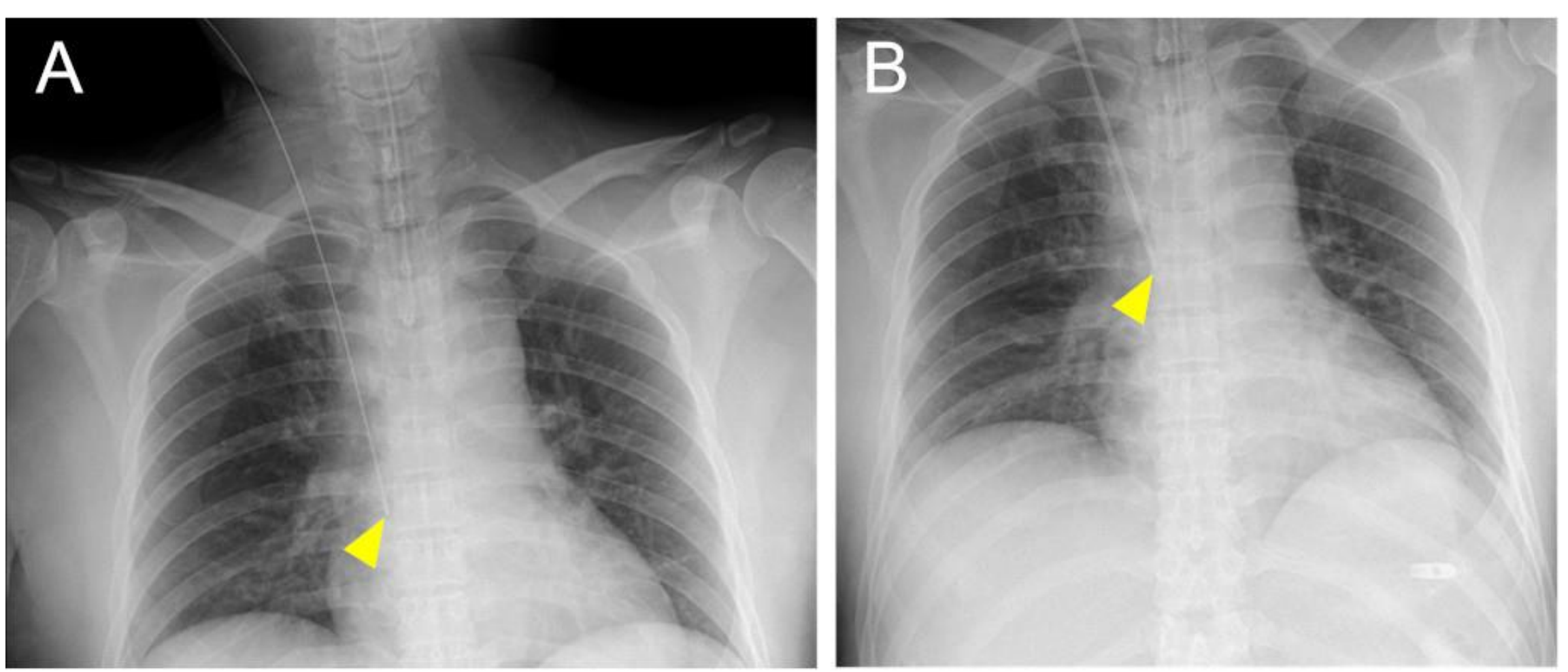


Fig. 4

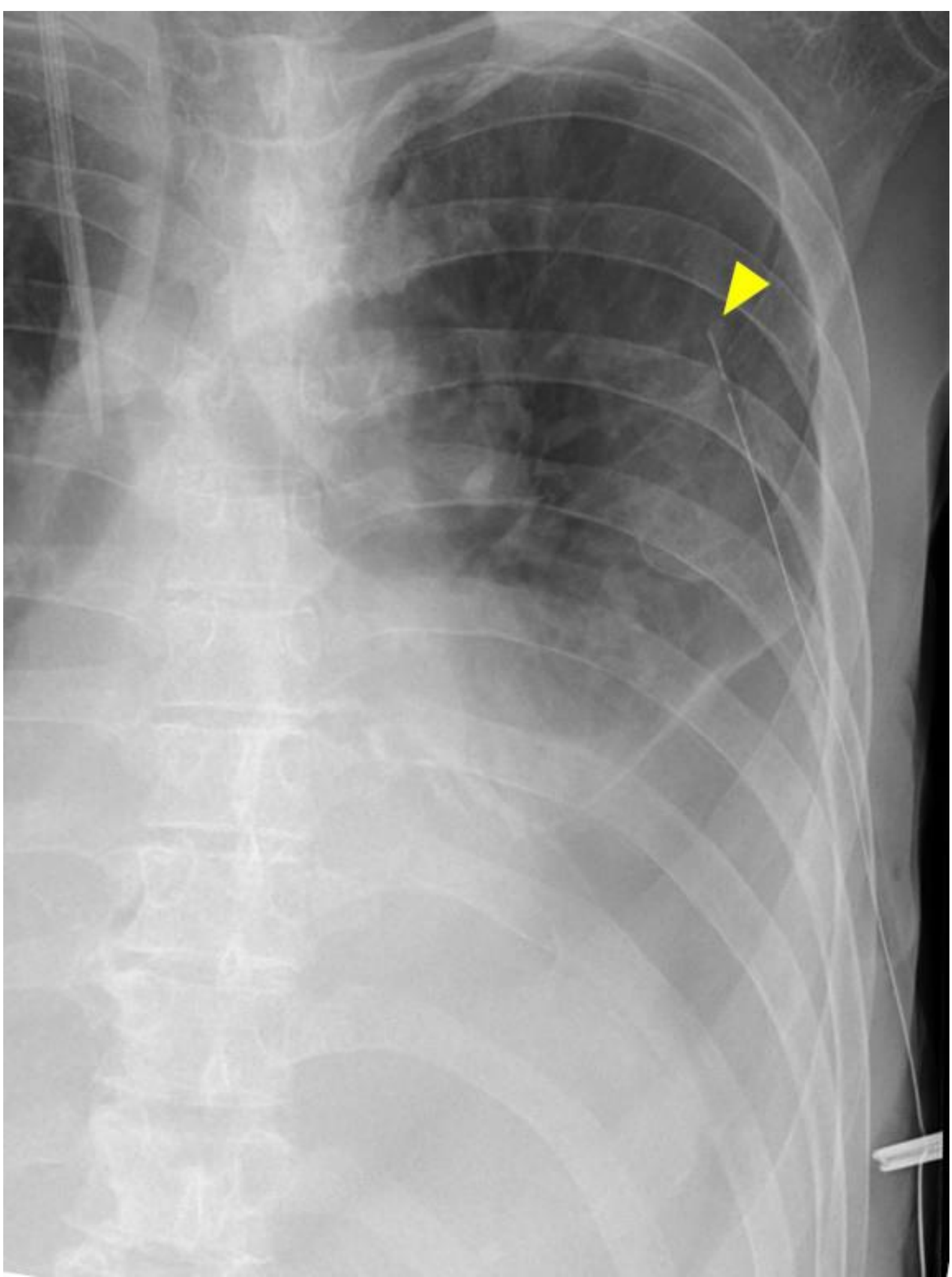


Fig. 5

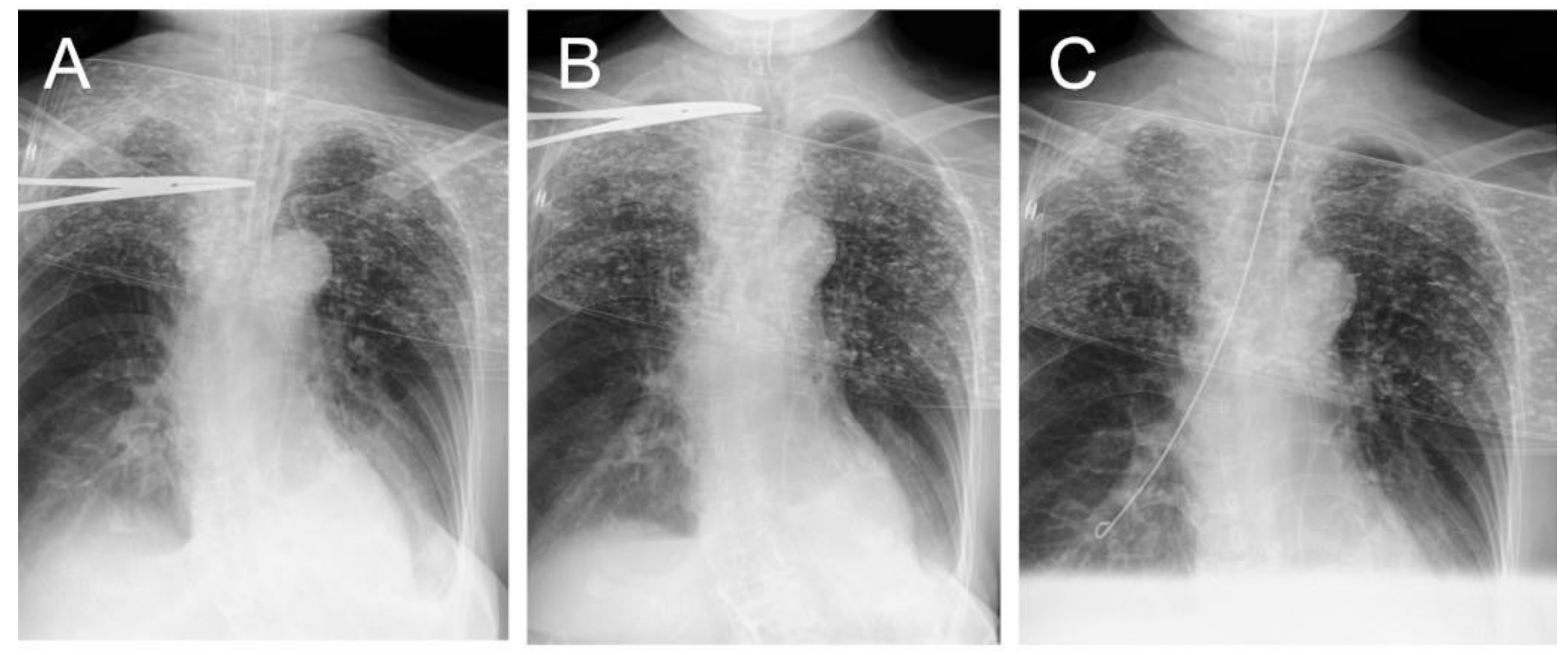


Fig. 6

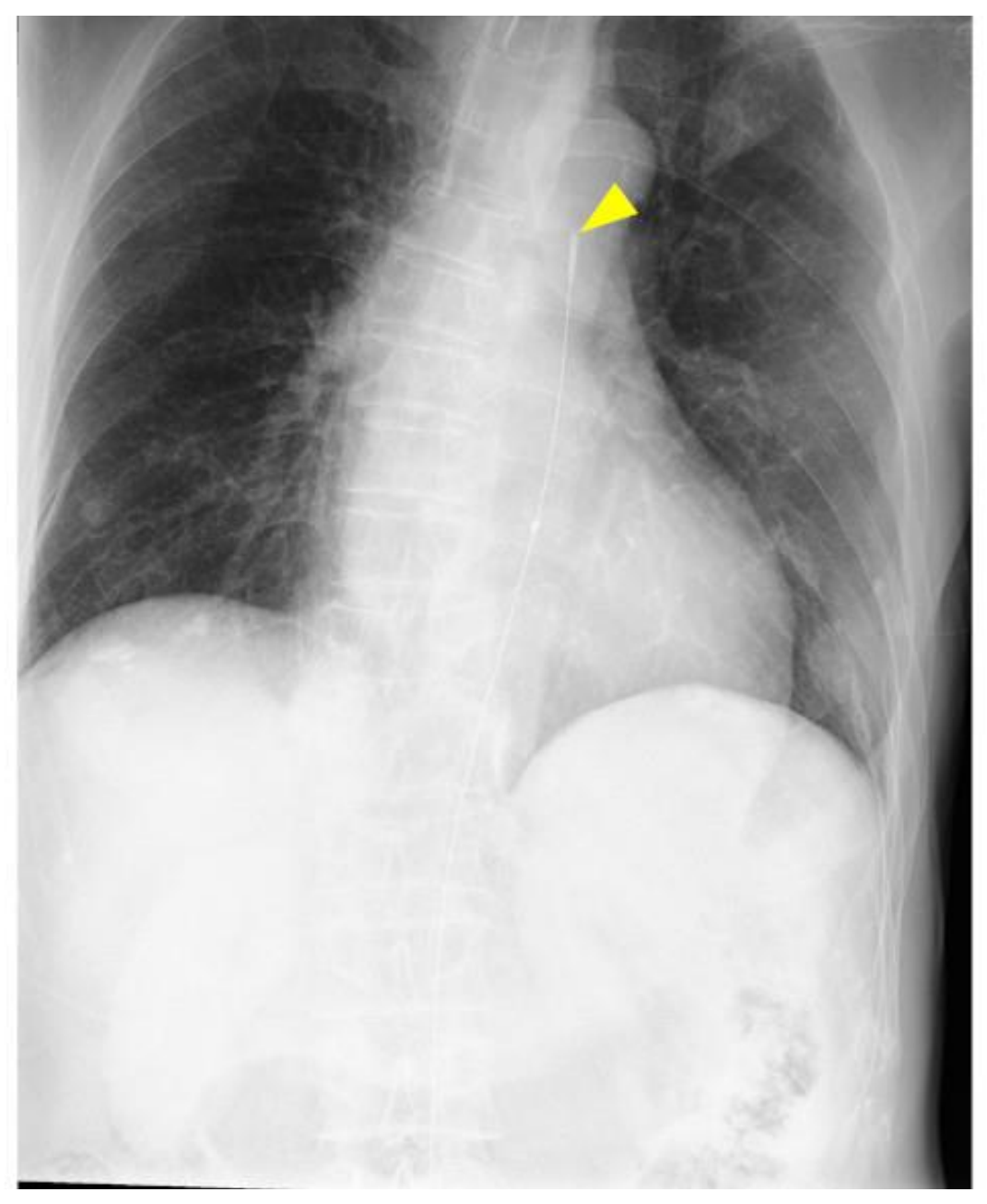


Fig. 7

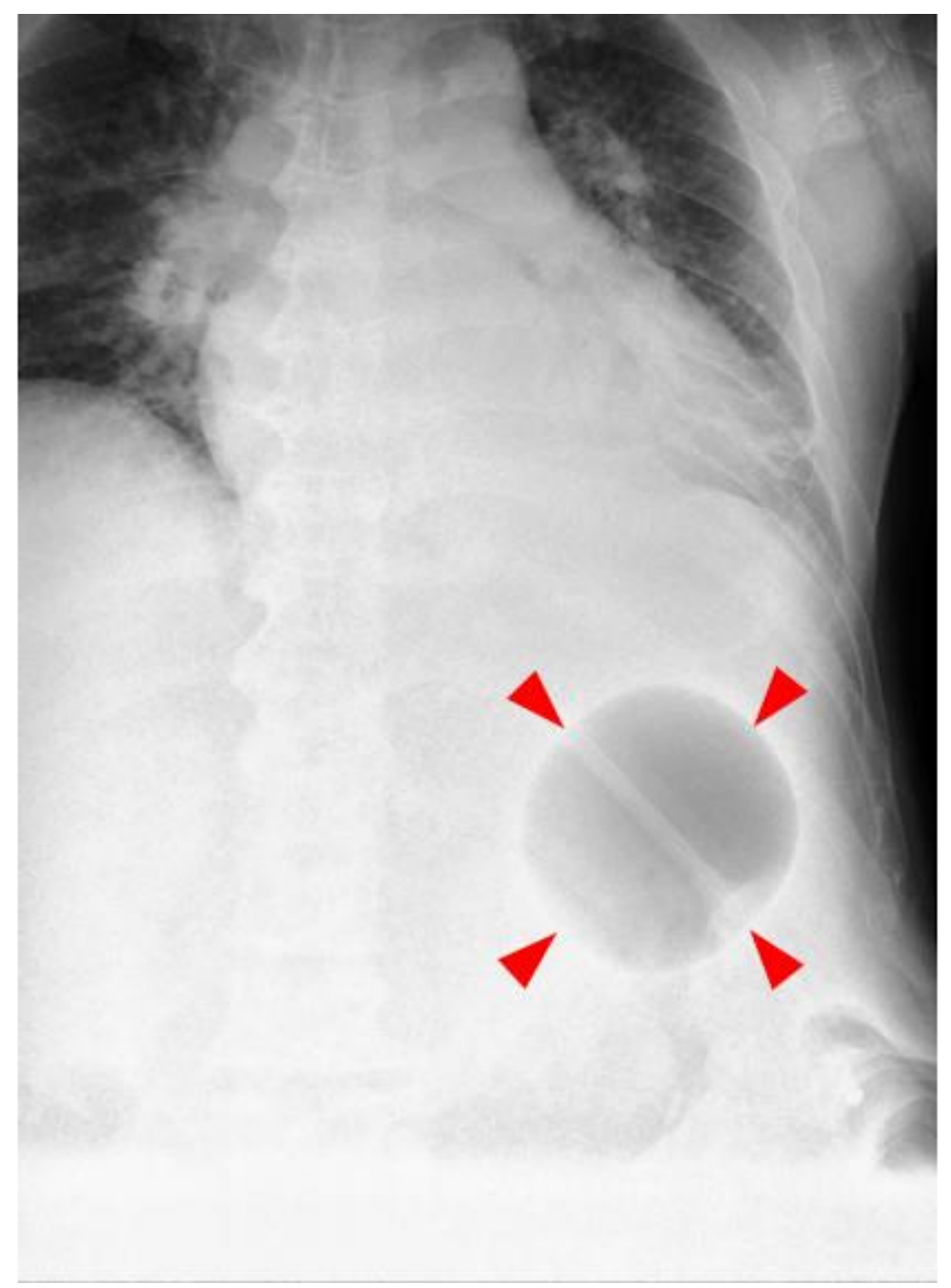

